\title{
Experimental determination of cotton yarn strength at different speeds of movement obtained by various technological methods
}

\author{
Karim Sultanov ${ }^{1 *}$, Sabida Ismailova ${ }^{1}$, Bakhodir Baymuratov ${ }^{2}$, and Shamsidin Tulanov ${ }^{2}$ \\ ${ }^{1}$ Institute of Mechanics and Seismic Stability of Structures, Uzbekistan Academy of Sciences, \\ Tashkent, Uzbekistan \\ ${ }^{2}$ Tashkent Institute of Textile and Light Industry, 100100 Tashkent, Uzbekistan
}

\begin{abstract}
Our results on the strength parameters of cotton yarns manufactured by carded and combed systems by ring and rotor spinning (CD-carded ring-spun, OE-carded rotor-spun, CM-combed ring-spun) are presented. Experiments to test yarns for strength were conducted on a "Statimat C" tensile installation at speeds of the lower end of the yarn from $100 \mathrm{~mm} / \mathrm{min}$ to $1000 \mathrm{~mm} / \mathrm{min}$. From the diagrams of yarn stretching to breaking, the changes in breaking load, specific breaking load, critical strain values at which the yarn breaks, time of stretching before breaking, depending on the strain rate for the considered types of yarns, were determined. The analysis of the experimental results obtained shows the quantitative and qualitative characteristics of the change in strength parameters of three varieties of yarns.
\end{abstract}

\section{Introduction}

In modern technical processes of textile production, the speed of movement of textile threads and yarns is increasingly increasing. With the need to increase the production of textiles, from the other side, the yarn breakage is also increased [1-4]. This material requires the study of the strength of cotton yarn at high speeds of its movement in technical processes of textile production.

Experimental studies of the strength of textile threads and yarns at such speeds of movement of the thread are practically absent. The results of experiments on the stretching of a carbon thread at speed of movement from 5 to $50 \mathrm{~m} / \mathrm{min}$ are presented [5]. Due to the lack of the possibility of carrying out experiments for determining the strength of textile threads and yarns at high speeds of their movement, there is a second way to investigate this problem-theoretical [6].

With the known laws of change of external forces by methods of mathematical modeling of the thread movement, it is possible to determine the mechanisms of formation of the stress-strain state of textile threads and yarns in the whole technological process, to follow, to determine the strength and to establish the reasons for their breakage. For this, first of all, it is necessary to have a good law of deformed yarn during stretching. On the

\footnotetext{
*Corresponding author: sultanov.karim@mail.ru
} 
basis of serial experiments on the stretching of cotton yarns to rupture, an elastic-viscousplastic law of deformation was developed [6].

One of the main qualities of cotton yarns (hereinafter referred to as yarn) is their strength. It is known that yarns made of cotton fibers have a complex structure [7-11]. This structure plays a determining role in ensuring its strength. Therefore, the main goal of the technological processes of yarn manufacture is to ensure its maximum possible strength. Consequently, the yarn is directly related to the technology of its manufacture $[9,11]$.

\section{Materials and Methods}

Experiments to determine the strength of cotton yarns were conducted on the "Statimat C" installation in the "Centexuz" certification laboratory of the Tashkent Institute of Textile and Light Industry. The methodology for conducting experiments, the issues of their accuracy and methods of experimental data processing are given in [12-16].

Consider yarns manufactured by carded and combed systems using ring and rotor spinning methods. The yarns produced by these methods are of different structures and mechanical characteristics; therefore, they have different strength characteristics. Varieties of yarn spinning technology and their advantages and disadvantages are described in detail in $[9-11]$.

The yarns are made of medium staple cotton of the 1st grade, 1st maturity group, 4th fiber type with a nominal linear density of $T=29.0$ tex. The actual linear density of the yarn was determined anew each time before the experiment on stretching. Deviation from nominal linear density is less than $2 \%$, which is within the range of standard norms.

Three types of cotton yarns selected, differing in technological methods of manufacture, were tested for strength under tensile loads uniformly varying until the yarn breakage. The "Statimat C" installation stretches yarn samples at different speeds. This is in fact equivalent to the different strain rates of the yarn under stretching.

There is a relationship between the speed of yarn motion and its strain rate (1):

$$
\varepsilon_{v}=\frac{d \varepsilon}{d t}=\frac{\vartheta}{L_{0}}
$$

where, $\varepsilon_{\vee}$ - is the strain rate of the yarn under stretching; $\varepsilon$ - is the longitudinal strain; $\vee$ - is the speed of motion of the lower end of the yarn under stretching; and $\mathrm{L}_{0}$ - is the base length of the yarn under stretching.

In experiments conducted on the installation, it is possible to vary the speed of motion $\vee$ within the range of $100<\mathrm{V}<1000 \mathrm{~mm} / \mathrm{min}(0.0017<\mathrm{V}<0.017 \mathrm{~m} / \mathrm{s})$. When stretching the yarn, the speed of motion was 100, 200, 300, 400, 500, 600, 700, 800, 900, $1000 \mathrm{~mm} / \mathrm{min}$. Each variety of yarns was tested 50 times at these values of the stretching speed before breaking; a total of 500 experiments were conducted for 10 values of speed. Typical tension diagrams of carded rotor spinning yarns are given in $[12,13,15]$.

\section{Results and Discussion}

In the experiments, the basic length of the yarn under tension in all the cases under consideration remained constant, $\mathrm{L}_{0}=500 \mathrm{~mm}$. The yarn strain rate at a speed $\mathrm{V}=1000$ $\mathrm{mm} / \mathrm{min}$ was $\varepsilon_{\vee}=0.033 \mathrm{~s}^{-1}$, and at $\vee=100 \mathrm{~mm} / \mathrm{min}$, it was $\varepsilon_{\vee}=0.0033 \mathrm{~s}^{-1}$, with 10 times difference. The value of the strain rate was determined by the formula (1) and by the values of the critical strain $\varepsilon_{\mathrm{k}}$, the stretching time of the yarn $\mathrm{t}_{\mathrm{k}}$ before breaking was determined by the following formula $\varepsilon_{\vee}=\varepsilon_{\mathrm{k}} / \mathrm{t}_{\mathrm{k}}$. The values of the parameters of breaking load $\mathrm{F}_{\mathrm{k}}$ and 
breaking strain $\varepsilon_{\mathrm{k}}$ for three varieties of yarn considered were determined from the diagram of the yarn tension $\mathrm{F}(\varepsilon)$ to breaking; they are shown in Table 1.

Table 1. Comparative values of $F_{k}$ and $\varepsilon_{k}$ for yarns with a linear density of $T=29.0$ tex manufactured by different production technologies

\begin{tabular}{|c|c|c|c|c|c|c|}
\hline \multirow{2}{*}{ Parameters } & \multicolumn{2}{|c|}{ OE yarn } & \multicolumn{2}{c|}{ CD yarn } & \multicolumn{2}{c|}{ CM yarn } \\
\cline { 2 - 7 } & $\begin{array}{c}\mathrm{V}=\mathbf{1 0 0} \\
\mathbf{m m} / \mathbf{m i n}\end{array}$ & $\begin{array}{c}\mathrm{V}=\mathbf{1 0 0 0} \\
\mathbf{m m} / \mathbf{m i n}\end{array}$ & $\begin{array}{c}\mathrm{V}=\mathbf{1 0 0} \\
\mathbf{m m} / \mathbf{m i n}\end{array}$ & $\begin{array}{c}\mathrm{V}=\mathbf{1 0 0 0} \\
\mathbf{m m} / \mathbf{m i n}\end{array}$ & $\begin{array}{c}\mathrm{V}=\mathbf{1 0 0} \\
\mathbf{m m} / \mathbf{m i n}\end{array}$ & $\begin{array}{c}\vee=\mathbf{1 0 0 0} \\
\mathbf{m m} / \mathbf{m i n}\end{array}$ \\
\hline$F_{k}^{\max }, \mathrm{cN}$ & 317.53 & 322.13 & 441.0 & 464.97 & 512.91 & 539.18 \\
\hline$\varepsilon_{k}^{\mathrm{max}}, \%$ & 7.73 & 7.93 & 8.23 & 8.06 & 8.89 & 8.21 \\
\hline$F_{k}^{\min }, \mathrm{cN}$ & 192.42 & 216.72 & 294.88 & 338.22 & 399.3 & 408.82 \\
\hline$\varepsilon_{k}^{\min }, \%$ & 5.45 & 5.86 & 6.67 & 6.51 & 7.13 & 6.72 \\
\hline$F_{k}^{c p}, \mathrm{cN}$ & 265.93 & 275.82 & 377.78 & 407.03 & 463.75 & 474.87 \\
\hline$\varepsilon_{k}^{c p}, \%$ & 6.85 & 7.09 & 7.58 & 7.44 & 8.21 & 7.59 \\
\hline
\end{tabular}

The value of the critical tensile force at the time of yarn breaking, which is a strength factor as well as the specific breaking load $\mathrm{P}_{\mathrm{p}}=\mathrm{F}_{\mathrm{k}} / \mathrm{T}$ for all three varieties of yarn considered, increases slightly with an increase in the speed of motion (Table 1).

This fact confirms the well-known property of deformable materials - with an increase in the strain rate, the resistance of the material to the effect of external loads increases, therefore, the material exhibits greater strength. The value of the critical strain at which the yarn breaks does not always increase with an increase in the speed of motion (Table 1). This fact means that the change in the strain rate does not affect the values of $\varepsilon_{\mathrm{k}}$.

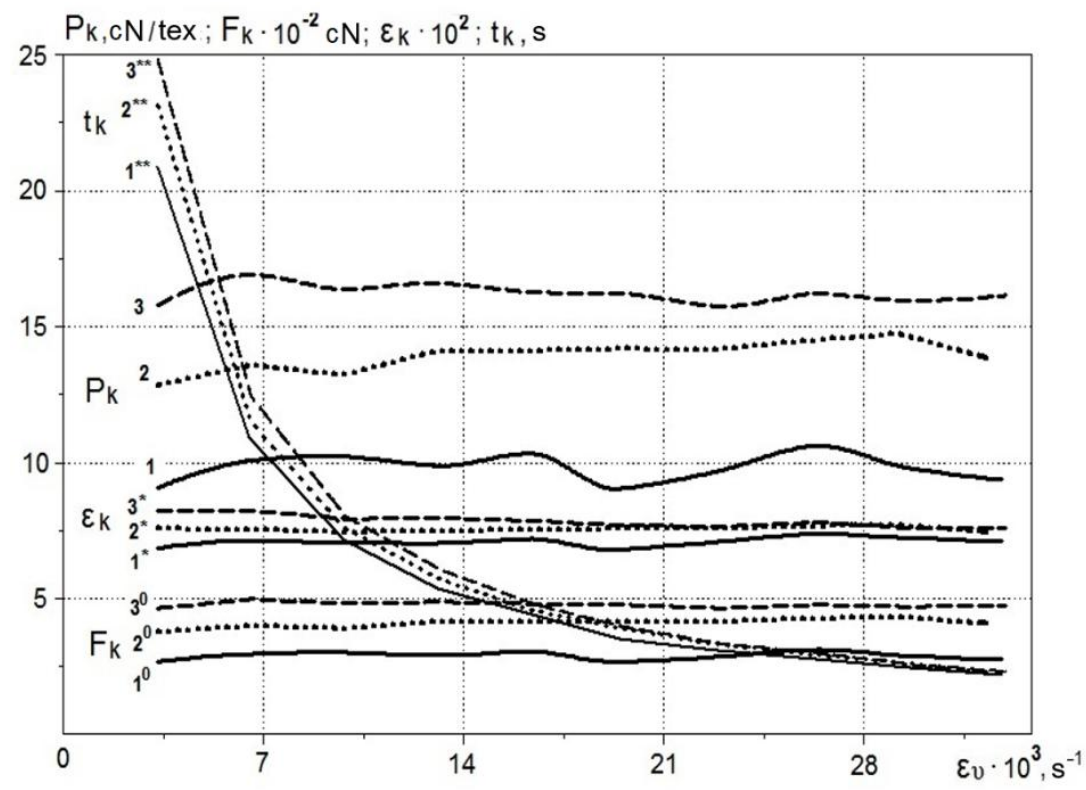

Fig. 1. Dependences of the strength parameters of the yarns on the strain rate under tension to breakage: carded rotor spun yarn (solid line curves), carded ring spun yarn (dashed-line curves), combed ring spun yarn (dotted curves) 
Fig. 1 shows the changes in strength parameters depending on the strain rate for the considered types of cotton yarns. Here, the pattern of the change in parameters $F_{k}, P_{k}, \varepsilon_{k}, t_{k}$ and $\varepsilon_{\vee}$ is evident. 1 curve refers to carded rotor yarns (solid lines), 2 curve refers to carded ring yarns (dashed-line curves) and curves 3 refer to combed ring yarns (dotted curves). Curves 1-3 refer to a change in the specific breaking load (strength) $P_{k}$, curves $1^{0}-3^{0}$ refer to a change in breaking load $F_{k}$, curves $1^{*}-3^{*}$ refer to a change in critical strain $\varepsilon_{\mathrm{k}}$, curves $1^{* *}-3^{* *}$ refer to a change in the time of yarn stretching to a breakage $t_{k}$. The values of $F_{k}$ and $\varepsilon_{\mathrm{k}}$ along the ordinate axis are determined with a factor of $10^{2}$ and $10^{-2}$, respectively.

As seen from Fig. 1, the strength parameters $F_{k}, P_{k}$, and $\varepsilon_{k}$ for all types of yarns, for the values of the strain rate variation from $0.0033 \mathrm{~s}^{-1}$ to $0.033 \mathrm{~s}^{-1}$, remains practically constant. With an increase in the strain rate, a slight increase in the strength of the carded ring yarn is observed (dashed-line curve 2, Fig. 1). The values of the parameters $\mathrm{F}_{\mathrm{k}}, \mathrm{P}_{\mathrm{k}}$, and $\varepsilon_{\mathrm{k}}$ for different types of yarns at all values of the strain rates are quantitatively different, while the highest values of these parameters are observed for the combed ring yarns (curves 3 ). The yarn produced by the carded system in the ring method has average values of strength parameters (curves 2), and the carded rotor spun yarn has the least value of strength parameters (curves 1).

In the case of carded ring yarns, the breaking load $F_{k}$ and specific breaking load $\mathrm{P}_{\mathrm{k}}$ are approximately $30 \%$ higher than these values obtained for carded rotor yarns. The yarn manufactured by the combed system in the ring method, as expected, has a $15-20 \%$ higher strength factor than in the second case. The critical strain values at which the yarn breaks remain approximately unchanged.

It is characteristic that in all cases with an increase in the speed of yarn motion, i.e. the strain rate under tension, the time of the yarn strain from the beginning to a breakage $t_{k}$ is substantially reduced. The value of $t_{k}$, as well as the value of the strain rate, decreases by approximately 10 times. The change in the yarn speed affects the values of $t_{k}$ in a tangible way. This means that even at low strain rates, the tensile dynamic properties of the yarn are substantial.

The change in the parameter $t_{k}$ occurs approximately according to an exponential law with an increase in the strain rate and already at $\varepsilon_{\vee}=0.033 \mathrm{~s}^{-1}$ approaches its limit value. Hence, it follows that within the strain rate of $\varepsilon_{\vee}=0.0033 \div 0.033 \mathrm{~s}^{-1}$, the dynamics of the yarn strain process is fully manifested. A further increase in $\varepsilon_{\vee}$ affects the yarn strength less intensely. Judging by the changes in $t_{k}\left(\varepsilon_{v}\right)$ (Fig. 1), it can be assumed that $t_{k}$ is 1 second. Starting from a strain rate $\varepsilon_{\mathrm{V}}=0.015 \mathrm{~s}^{-1}$, where $\mathrm{t}_{\mathrm{k}}=5 \mathrm{~s}$, the value of $\mathrm{t}_{\mathrm{k}}$ slowly decreases and, presumably, at $\varepsilon_{\vee}=0.05 \div 0.1 \mathrm{~s}^{-1}$ reaches its limit value of $1 \mathrm{~s}$. This result should be taken into account when constructing theoretical models of cotton yarn strain.

The effect of the tensile strain rate on the strength characteristics was discussed above for yarns with an actual linear density of $T=29.0$ tex. The influence of the methods of cotton yarn manufacture on its strength parameters was also considered. The strength characteristics of yarns produced using different spinning systems also depend on their linear densities, which is the subject of future research.

\section{Conclusions}

The changes in strength parameters $F_{k}, P_{k}, \varepsilon_{k}, t_{k}$ and $\varepsilon_{\vee}$ were experimentally determined for the yarns produced by carded and combed spinning systems by ring and rotor spun methods. It was determined that within the range of strain rate variation from $0,0033 \mathrm{~s}^{-1}$ to $0,033 \mathrm{~s}^{-1}$, the values of the strength parameters $F_{k}, P_{k}$, and $\varepsilon_{k}$ for each variety of yarns remain constant.

It was determined that the values of breaking load $F_{k}$ and specific breaking load $\mathrm{P}_{k}$ differ according to the method of manufacturing. The highest values are observed for 
combed ring yarns, and the least values are observed for carded rotor spun yarns. The values for carded yarns manufactured by the ring-spun method lie in between.

The critical strain values $\varepsilon_{\mathrm{k}}$ remain constant at all strain rates and do not depend on the yarn production methods. The values of yarn strain time, i.e., the time from the beginning of stretching to breakage $t_{k}$ depend substantially on the strain rate even at its low limits. With an increase in the strain rate, the value of $t_{\mathrm{k}}$ decreases exponentially and at $\varepsilon_{\mathrm{V}}=0.01 \mathrm{~s}^{-1}$ reaches its limit of $t_{k}=1 \mathrm{sec}$ and then remains constant. The value of $t_{k}$ is practically independent of the yarn production methods. With all three yarn production methods, the value of $t_{\mathrm{k}}$ remains approximately the same.

\section{References}

1. A. Grechukhin, V. Seliverstov, P. Rudovskiy, Journal of the Textile Institute 108(12), 2067-2072 (2017)

2. J. Gafurov, B. Mardonov, M. Peerzada, K. Gafurov, Fibers\&Textiles in Eastern Europe 2(110), 19-27 (2015)

3. J. Gafurov, B. Mardonov, M. Peerzada, Science International. Lahore 24(1), 55-58 (2012)

4. Zh. Gafurov, B. Mardonov, Problemy mekhaniki 2, 15-19 (2012)

5. A. Jounes, V. Sonkarn, A. Seidel, M. Waldmann, Ch. Cherif, J. Hausding, Textile Research Journal 82(7), 685-699 (2012)

6. W. Morton, J. Hearle, Physical properties of textile fibers, 765 (Cambridge Press, London, 2008)

7. V. Shcherbakov, Applied and structural mechanics of fibrous materials, 304 (Tisoprint Press, Moscow, 2013)

8. G. Kukin, A. Solov'ev, Textile materials science, 380 (Light industry Press, Moscow, 1964)

9. T. Kukin, A. Solovyov, A. Koblyakov, Textile materials science (fibers andyarns), 350 (Legprombitizdat Press, Moscow, 1989)

10. V. P. Shcherbakov, N. S. Skulanova, Fundamentals of the theory of deformation and strength of textile materials, 332 (Kosygin Moscow State Technical University Press, Moscow, 2008)

11. S. Ismailova, K. Sultanov, Mechanics of Solids 5, 578-592 (2015)

12. K. Sultanov, S. Ismoilova, Journal of Fashion Technology \& Textile Engineering 10(3), 11 (2017)

13. K. Sultanov, S. Ismoilova, N. Mamatova, Journal of Fashion Technology \& Textile Engineering 6(1), 10 (2018)

14. K. Sultanov, S. Ismailova, Sh. Tulanov, Izvestiya Vuzov. Technology of the textile industry 5(365), 109-115 (2016)

15. S. Ismailova, K. Sultanov, Sh. Tulanov, Izvestiya Vuzov. Technology of the textile industry 4 (364), 63-67 (2016)

16. K. Sultanov, S. Ismailova, Izvestiya Vuzov. Technology of the textile industry 3(375), 95-101 (2018) 